Arq. Bras. Med. Vet. Zootec., v.69, n.5, p.1251-1258, 2017

\title{
Cartogenina associada à microperfuração do osso subcondral na reparação da cartilagem articular em equinos
}

[Kartogenin associated to micro of the subchondral bone in the joint cartilage repair in horses]

\author{
S.S. Rocha Junior ${ }^{1}$, D.S.F. Ribeiro ${ }^{1}$, M.P.A. Lima ${ }^{1}$, R.C. Valadares ${ }^{1}$, E. Pimenta ${ }^{1}$, \\ J.F. Magalhães ${ }^{1}$, J.C. Camillo ${ }^{1}$, O. Spadeto Júnior ${ }^{1}$, R.R. Faleiros ${ }^{2,3^{*}}$
}

\footnotetext{
${ }^{1}$ Aluno de pós-graduação - Escola de Veterinária - Universidade Federal de Minas Gerais - Belo Horizonte, MG

${ }^{2}$ Escola de Veterinária - Universidade Federal de Minas Gerais - Belo Horizonte, MG

${ }^{3}$ Bolsista de produtividade do $\mathrm{CNPq}$
}

\begin{abstract}
RESUMO
A reconstituição da cartilagem articular danificada por doenças de desenvolvimento, trauma e osteoartrite tem sido um grande desafio na veterinária. O objetivo do presente estudo foi avaliar o uso da cartogenina, uma molécula capaz de induzir diferenciação de células mesenquimais em condrócitos, associado ou não à técnica de microfratura na reparação de defeitos condrais induzidos na tróclea femoral de equinos. Foram utilizados seis equinos pesando em média ( \pm DP) $342 \pm 1,58 \mathrm{~kg}$, com idade de 7,2 $\pm 1,30$ anos e

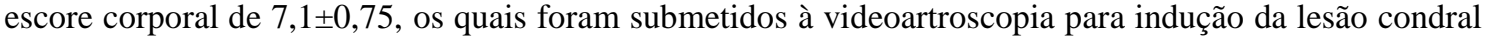
de $1 \mathrm{~cm}^{2}$ na tróclea lateral do fêmur e à realização da técnica de microperfuração do osso subcondral de ambos os joelhos. Foram realizadas quatro aplicações semanais com $20 \mu \mathrm{M}$ de cartogenina intra-articular em um dos joelhos (grupo tratado) e solução de ringer com lactato na articulação contralateral (grupo controle). Os animais foram submetidos a avaliações física, radiográfica, ultrassonográfica, por um período de 60 dias. Não houve qualquer diferença estatística entre as articulações tratadas e as controle. A terapia com cartogenina, segundo protocolo utilizado, não produziu melhora clínica em lesões osteocondrais induzidas e tratadas com microperfurações na tróclea lateral do fêmur em equinos.
\end{abstract}

Palavras-chave: cavalo, cartilagem hialina, osteoartrite, ultrassonografia, radiografia

\begin{abstract}
Articular cartilage reconstruction is still a challenge in Veterinary Medicine. The aim was to evaluate the therapeutic effects of kartogenin, a small molecule that promotes chondrocyte differentiation, in the repair of induced chondral defects pretreated with subchondral drilling. Six horses with a mean $( \pm S D)$ weight of $342 \pm 1.58 \mathrm{Kg}$, aging $7.2 \pm 1.30$ years, and with a mean $7.1 \pm 0.75$ body score condition were used. In both stifles, a $1 \mathrm{~cm}^{2}$ chondral defect was induced in the lateral femoral trochlea followed by treatment with subchondral drilling. Four intra-articular injections with kartogenin (20mM) were performed weekly in one stifle (treated group). The same procedure using saline solution was performed in the contralateral joint (control group). Clinical, radiographic, ultrasound evaluations were performed for a period of 60 days. No statistical differences were detected between groups in any of the studied variables. Although kartogenin has shown to improve articular cartilage repair in laboratory animal models, the same was not observed in this equine model. In conclusion, the kartogenin therapy, according to the used protocol, did not promote any clinical benefit in equine femoral trochlear defects pretreated with subcondral drilling.
\end{abstract}

Keywords: horse, hyaline cartilage, osteoarthritis, ultrasound, radiography

Recebido em 25 de julho de 2015

Aceito em 13 de junho de 2016

*Autor para correspondência (corresponding author)

E-mail: faleirosufmg@gmail.com 


\section{INTRODUÇÃO}

A reconstituição da cartilagem articular danificada por doenças de desenvolvimento, trauma e osteoartrite tem sido um grande desafio, tanto na medicina humana quanto na veterinária. Entre as enfermidades articulares dos equinos, a osteoartrite (OA) é a mais frequente, correspondendo a $60 \%$ das causas (McIlwraith, 1996). A OA é definida como um processo crônico caracterizado por progressiva deterioração da cartilagem articular, acompanhado por alterações na cápsula articular, no osso subcondral e nos demais tecidos articulares (McIlwraith,1996; Brandt et al., 2009). A principal consequência da OA é a degradação da cartilagem articular que, por sua vez, apresenta importantes limitações quanto ao seu potencial de regeneração (Hurtig et al., 1988).

Uma vez degradada a cartilagem articular, essa não possui a capacidade de autorregeneração. Nos casos em que há perda de cartilagem, com exposição do osso subcondral, ocorrerá a formação de um tecido cicatricial fibroso, podendo ou não sofrer metaplasia para fibrocartilagem (Hurtig et al., 1988). Para contornar o potencial limitado de regeneração, pesquisas vêm sendo realizadas usando terapias celulares como o plasma rico em plaquetas e células-tronco mesenquimais. Mesmo com esses avanços, o tecido cicatricial predominantemente alcançado é a fibrocartilagem e não a cartilagem hialina (Milano et al., 2010; McIlwraith et al., 2011; Yamada et al., 2011; Milano et al., 2012).

Recentemente, demonstrou-se que uma molécula denominada cartogenina tem a capacidade de diferenciar células-tronco mesenquimais em condrócitos in vitro (Johnson et al., 2012). Também se demonstrou que seu uso pode melhorar a condição clínica de animais de laboratório com defeito condral induzido (Johnson et al., 2012; Xu et al., 2014).

Devido ao potencial do uso da cartogenina para promover regeneração condral e pelo fato de ainda não existirem pesquisas utilizando equinos como animais de experimentação, objetivou-se fazer análise clínica, radiográfica, ultrassonográfica do líquido sinovial de equinos submetidos ao tratamento com cartogenina, associado ou não à técnica de microfratura, para o reparo de defeitos condrais induzidos. A hipótese é a de que a aplicação intra-articular de cartogenina produz melhora clínica em equinos com defeito condral induzido pré-tratado com microperfurações subcondrais.

\section{MATERIAL E MÉTODOS}

O estudo experimental foi conduzido atendendo a todos os requisitos do Comitê de Ética em Experimentação Animal (CEUA/UFMG), sob protocolo de número $\mathrm{n}^{\circ}$. 36/2014. Foram utilizadas cinco éguas e um cavalo, adultos, sem raça definida, com peso médio $( \pm \mathrm{DP})$ de $342 \pm 1,58 \mathrm{~kg}$, com a idade de 7,2 $\pm 1,30$ anos e escore corporal de 7,1 $\pm 0,75$. Todos os animais foram provenientes da Fazenda Modelo de Pedro Leopoldo - MG e foram considerados clinicamente normais após exames físico e laboratorial (hemograma e perfis hepático e renal). Nenhum dos equinos tampouco apresentou alterações radiográficas e ultrassonográficas das articulações femorotibiopatelares.

Os animais foram mantidos em baias individuais de alvenaria, por período de adaptação de 30 dias, recebendo diariamente água, feno de tifton e sal mineral ad libitum e ração comercial (Essence, Presence Nutrição Animal Ltda., Brasil) na quantidade equivalente a $1 \%$ do peso vivo, dividida em duas porções.

Utilizou-se um delineamento experimental do tipo blocos ao acaso, comparando-se grupo tratado versus grupo controle em diferentes tempos. Para tanto, lesões condrais foram induzidas em ambas as articulações femoropatelares de cada animal, sendo uma articulação controle e outra tratada. Para cada grupo, havia três articulações do lado direito e seis do lado esquerdo.

O primeiro procedimento artroscópico para indução da lesão e realização das microperfurações foi considerado o tempo 0 (T$0)$. Os animais foram mantidos em decúbito dorsal, e na articulação femorotibiopatelar foram criados dois portais de $1 \mathrm{~cm}$. O primeiro portal foi criado entre os ligamentos patelares medial e intermédio. O segundo portal foi criado entre os ligamentos patelares intermédio e lateral, onde, por meio da abertura, foi introduzida uma cureta número 1. Com este instrumento, realizou-se 
uma lesão condral de $1 \mathrm{~cm}^{2}$ na porção distal da tróclea lateral. $\mathrm{O}$ defeito foi induzido preservando-se a integridade do osso subcondral em ambas as articulações.

Após completa exposição do osso subcondral, a mensuração dos lados da lesão foi realizada utilizando-se as pontas ativas da pinça Ferris Smith (Karl Storz), que se distanciam em $1 \mathrm{~cm}$ quando em abertura total. Feito isso, o osso subcondral foi perfurado com o auxílio de um pino de Steinmande $1,5 \mathrm{~mm}$ de diâmetro, acoplado a uma microrretífica. Foi feito um total de cinco perfurações por lesão, uma no centro e quatro nas extremidades. Os pinos eram aprofundados até que houvesse o extravasamento de glóbulos de gordura advindos da medula óssea. Tal procedimento foi realizado em todas as articulações.

Nas articulações do grupo tratado, aplicações intra-articulares de cartogenina foram realizadas ao final da primeira artroscopia e semanalmente por mais três semanas. Para tanto, previamente ao procedimento cirúrgico, preparou-se uma solução estoque adicionando-se $5 \mathrm{mg}$ de cartogenina (Kartogenin, Sigma-Aldrich $®$, USA) em 70mL de dimetil-sulfóxido (DMSO p.a $\geq$ $99,9 \%$, Sigma-Aldrich®, USA) em capela de fluxo laminar. Após a diluição, alíquotas contendo $1 \mathrm{mLda}$ solução foram separadas em tubos do tipo Eppendorf e imediatamente congeladas a $-20^{\circ} \mathrm{C}$.

Ao final da artroscopia e nos dias de tratamento, a solução de tratamento era preparada utilizandose $1 \mathrm{ml}$ da solução de estoque adicionada a $19 \mathrm{~mL}$ de ringer com lactato, obtendo-se concentração final de $20 \mu \mathrm{M}$ de cartogenina. Um volume igual de solução ringer lactato foi administrado como placebo nas articulações do grupo controle. As soluções foram sempre preparadas pela mesma pessoa e entregues ao cirurgião em uma seringa de $20 \mathrm{~mL}$ acoplada a um filtro de seringa de 0,20 micras, sem identificação e recoberta por esparadrapo, de forma que o estudo fosse conduzido de maneira cega durante todo o período experimental.

Os animais foram acompanhados durante $\mathrm{o}$ período de 60 dias, quando foram submetidos a exame físico diário e à avaliação de claudicação semanal, segundo a graduação preconizada pela Associação Americana de Médicos Veterinários de Equinos (AAEP, 1996). Além dessa avaliação, foi criada uma segunda escala de claudicação, visando a alterações na fase cranial da passada dos membros posteriores (Tab. 1).

Tabela 1. Graduação de claudicação, visando a alterações na fase cranial da passada

\begin{tabular}{cl}
\hline Grau & \\
\hline 0 & Não claudica \\
1 & Encurtamento da fase cranial da passada \\
2 & Encurtamento da fase cranial e de elevação da passada, claudicação evidente \\
3 & Dificuldade em caminhar e relutante em trotar \\
4 & Não apoia o membro no solo \\
\hline
\end{tabular}

As avaliações radiográficas foram realizadas antes e com 30 e 60 dias da indução lesional. Utilizaram-se projeções lateromedial oblíqua e caudocranial das articulações com exposição a $90 \mathrm{Kv}$ e $3,5 \mathrm{~mA}$, seguindo o sistema de classificação radiográfica estipulada por Crawford (1991).

As articulações femoropatelares também foram avaliadas semanalmente por meio de ultrassonografia, utilizando-se um aparelho de uso veterinário (M5, Mindray) munido de probe linear com frequência entre 7 e 10MHz. Para avaliação da superfície articular e da presença de erosões sobre o osso subcondral, utilizou-se o escore de graduação estipulado por (Larsen et al., 1977). Junto a essa avaliação, também foram mensuradas as áreas do recesso femoropatelar lateral e o recesso femorotibial medial, com o intuito de avaliar efusão articular.

Os dados paramétricos foram submetidos à análise de variância em blocos ao acaso considerando-se os efeitos de tempo, sendo as médias comparadas pelo teste de Tukey. A comparação entre grupos no mesmo tempo foi realizada pelo teste t de Student. Os dados não paramétrios foram analisados quanto ao efeito do tempo pelo teste de Friedman seguido pelo teste de Dunn, e quanto ao efeito do tratamento, pelo teste de Mann-Whitney. Para todos os testes, foi considerado o nível de significância de $\mathrm{P}<0,05$. 


\section{RESULTADOS}

Durante o procedimento artroscópico para realização da lesão, não se observaram quaisquer alterações prévias nas articulações utilizadas. A lesão condral na porção distal da tróclea lateral foi realizada de forma satisfatória. Em todos os procedimentos de microperfurações, gotas de gordura foram obtidas, demonstrando acesso à medula óssea.

Todos os animais comportaram-se bem no pósoperatório imediato e nos dias subsequentes, não sendo observadas reduções no consumo de feno e ração. Os equinos se mantiveram em estação na maior parte do dia, não havendo distribuição desigual de peso entre os membros posteriores

\section{Escore AAEP}

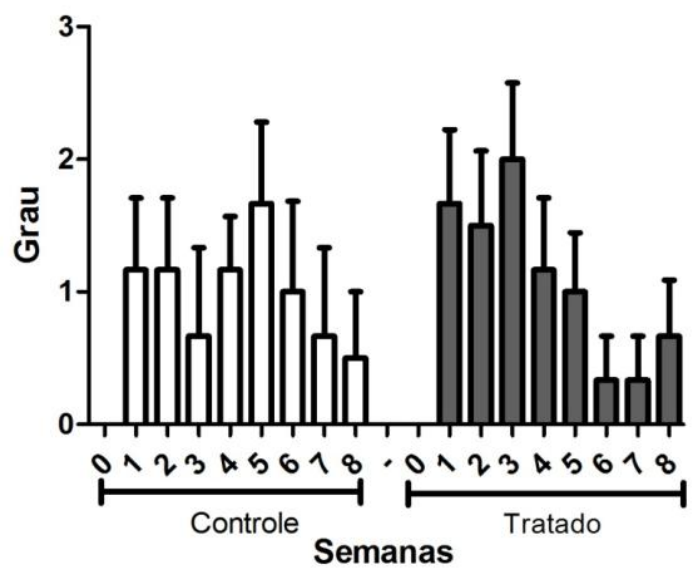

ou alteração em sua mobilidade, sendo apenas visualizada uma leve rotação lateral da articulação femorotibiopatelar, quando os animais eram submetidos ao trote.

Algum grau de claudicação foi observado em todos os animais nas semanas seguintes à cirurgia. Entretanto, houve predomínio de claudicações discretas. Segundo a classificação da AAEP (1996), considerando-se membros pélvicos direito e esquerdo, o grau médio de claudicação $( \pm \mathrm{DP})$ foi de $1,42 \pm 1,31$ na primeira semana e de $0,58 \pm 1,08$ na oitava semana. Não houve diferença estatística entre tempos ou grupos, considerando-se ambas as classificações para claudicação (Fig. 1).

Figura 1. Médias e erros-padrão da avaliação de claudicação segundo AAEP (1996) ou metodologia própria de equinos submetidos à lesão condral na tróclea lateral do fêmur, tratados ou não com cartogenina.

Pelo exame radiográfico da articulação femorotibiopatelar, não houve alterações detectáveis da superfície e do espaço articular. Apenas foram visualizados os pontos de perfuração realizados no osso subcondral durante a execução da técnica de microperfuração na tróclea lateral nos tempos 30 e 60 . Achados semelhantes foram encontrados na avaliação ultrassonográfica, em que também foram visualizados os locais das perfurações no osso subcondral.
Em relação às medidas dos recessos femoropatelar lateral e femorotibialmedial (Fig. 2), houve aumento significativo quanto à circunferência e à área. Isso ocorreu a partir da primeira semana pós-operatória, havendo regressão gradual com o passar das semanas (Fig. 3 e 4). Nenhuma diferença estatística foi detectada entre grupos em um mesmo tempo. Durante avaliação dos recessos lateral e medial, também foi encontrada proliferação sinovial (Fig. 2), que se manteve até o fim do estudo. 

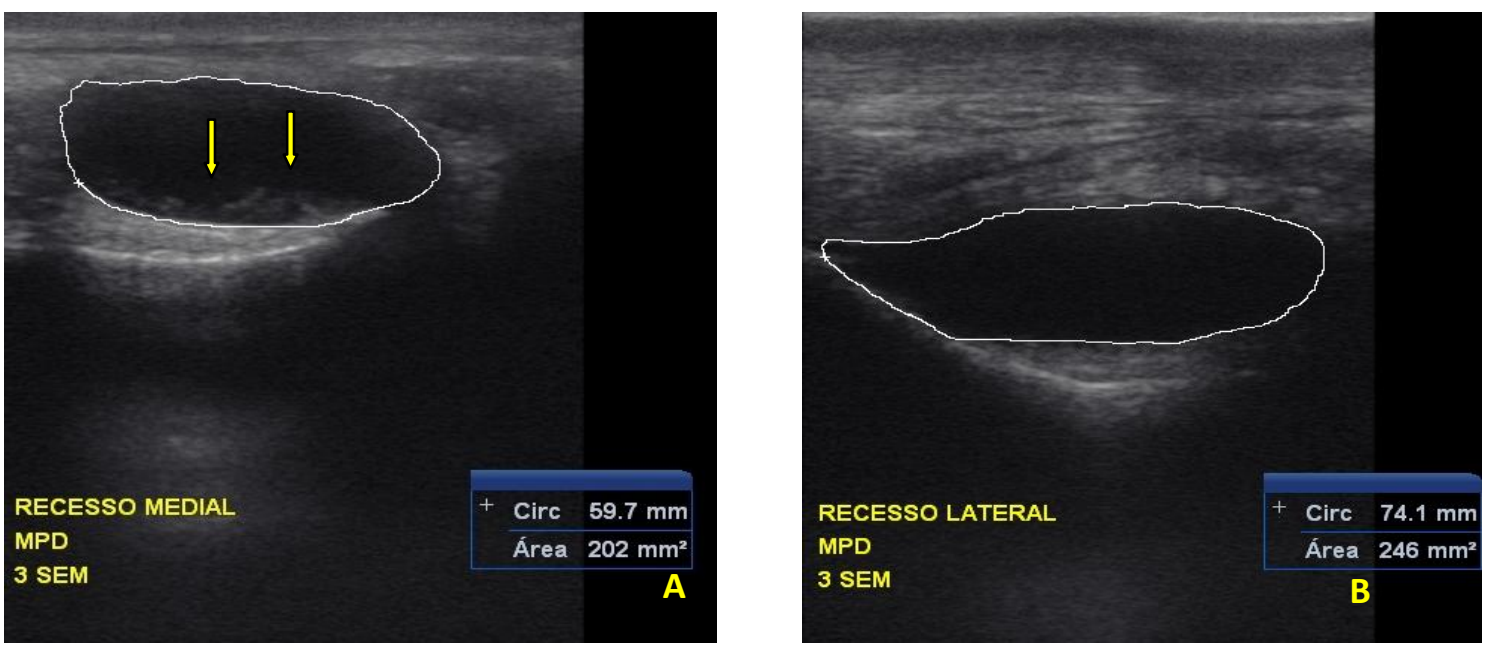

Figura 2. Imagem ultrassonográfica demonstrando a mensuração da circunferência e da área do recesso femorotibial medial de um equino submetido à lesão condral. Setas amarelas indicam proliferações sinoviais (A). Mensuração da circunferência e área do recesso lateral da articulação femoropatelar direita (B).
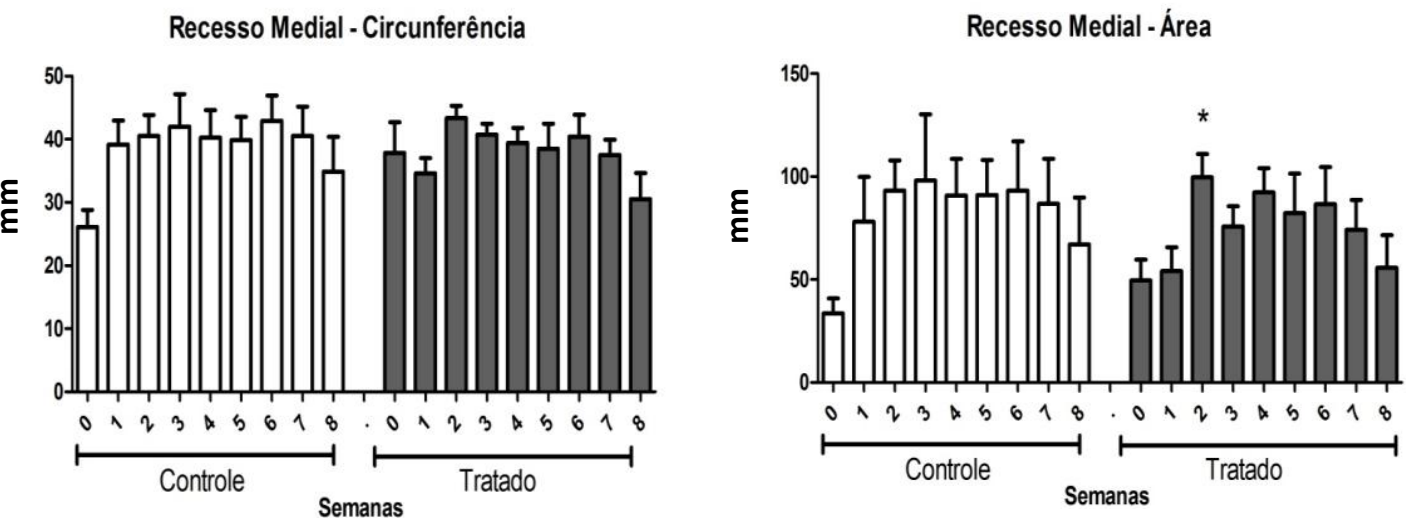

Figura 3. Médias e erros-padrão da circunferência e da área do recesso femoropatelar lateral de equinos submetidos à lesão condral na tróclea lateral do fêmur, tratados ou não com cartogenina.
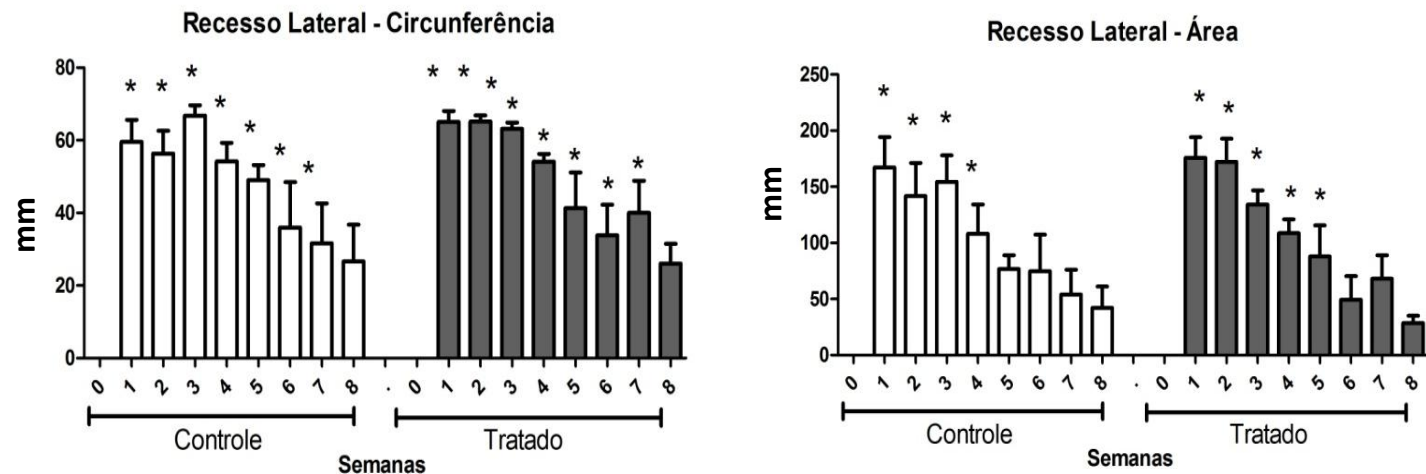

Figura 4. Médias e erros-padrão da circunferência e da área do recesso lateral da articulação femoropatelar de equinos submetidos à lesão condral na tróclea lateral do fêmur, tratados ou não com cartogenina. 


\section{DISCUSSÃO}

Como descrito por outros autores, que utilizaram modelos semelhantes de lesão condral no cavalo, claudicação leve era esperada no presente estudo. Escores semelhantes (1.3 \pm 0.08$)$ foram observados após lesões condrais induzidas de $1 \mathrm{~cm}^{2}$ no côndilo medial do fêmur (McIlwraith, 2009) e de $15 \mathrm{~mm}$ na crista troclear medial do fêmur (Frisbie et al., 2008). Esses achados de mesma intensidade dolorosa decorrente de lesões em diferentes locais da articulação femorotibiopatelar indicam influência predominante da exposição do osso subcondral. Sabe-se que, diferentemente da cartilagem articular, o osso subcondral é inervado, podendo haver sensibilização das fibras nervosas aferentes por mediadores inflamatórios como citocinas, prostaglandinas e óxido nítrico decorrentes do processo inflamatório instalado na articulação pela lesão (Werren e Grauw, 2010).

Referente à rotação do membro quando os animais eram submetidos ao trote, este achado foi semelhante ao encontrado por Yamada et al. (2011). Sugere-se que é uma adaptação postural ocasionada pela dor. E como não houve diferença entre as articulações, é possível inferir que a melhora gradual da claudicação foi ocasionada pela redução do processo inflamatório e não pelo efeito da cartogenina. Diferentemente do que foi encontrado com o uso de cartogenina em um modelo de osteoartrite em ratos (Johnson et al., 2012), a articulação que recebeu a molécula não apresentou melhor sustentação do peso comparado com a articulação controle.

No estudo radiográfico foi possível identificar, aos 30 e 60 dias, os pontos de microperfuração do osso subcondral no local da lesão. Entretanto, não houve alterações radiográficas típicas de lesão condral como a redução do espaço articular. Sabe-se, porém, que tais alterações só são evidentes quando há grande perda da cartilagem e mudança de $30-40 \%$ na densidade mineral óssea (Greenfield, 1986). Também não foram observadas lesões típicas de osteoartrite, resultado que difere de modelos em que o defeito foi realizado no côndilo femoral, quando foram descritas proliferações ósseas, formação de osteófitos, lise óssea e aumento da opacidade na região do defeito (McIlwraith et al., 2011).
Esses resultados radiográficos são condizentes com a claudicação discreta. Tomados em conjunto, esses achados demonstram que o modelo é bem tolerado pelos animais, sem trazer lesões ou sequelas que possam comprometer seu estado de bem-estar.

Ao exame ultrassonográfico, também foi possível documentar as microperfurações ósseas, demonstrando fácil acesso ao local da lesão. Este estudo, da mesma forma, foi importante para documentar e quantificar a efusão sinovial. Normalmente o recesso sinovial femorotibial medial possui uma certa quantidade de líquido sinovial (Swiderski et al., 2005), condizendo com a avaliação realizada no tempo zero. Quanto ao recesso femoropatelar lateral, este normalmente não é visualizado em cavalos normais (Denoix \& Coudry, 2008).

Com o processo inflamatório promovido pela cirurgia e pela indução da lesão, houve um aumento gradual na circunferência e na área do recesso femoropatelar lateral nas primeiras semanas subsequentes. Contudo, a efusão começou a regredir a partir da terceira semana, retornando a valores equivalentes aos basais a partir da quinta semana (Fig. 3). Alterações também foram verificadas no recesso femorotibial medial, com alterações discretas em suas dimensões (Fig. 4), entretanto com indícios de proliferação sinovial (Fig. 2). A presença de proliferações sinoviais durante as avaliações dos recessos é um achado comum e considerado inespecífico (Swiderski et al., 2005).

Independentemente da variável estudada e do tempo em que a análise foi realizada, não foram encontradas quaisquer diferenças entre o grupo tratado e o não tratado. Esses achados demonstram claramente que o uso da cartogenina não teve qualquer benefício clínico sobre as alterações articulares induzidas pela lesão condral. Estando de acordo com os achados encontrados por (Rocha Junior et al., 2016). Mas estes resultados estão em desacordo com estudos com osteartrite induzida por colagenase ou transecção de ligamentos no joelho de camundongos (Johnson et al., 2012), que verificaram melhora na porcentagem de apoio de peso nos membros tratados com cartogenina em relação ao membro controle. Ainda é prematuro discutir a discrepância dos resultados entre os dois modelos, contudo existem marcadas 
diferenças na biomecânica da articulação do joelho entre as espécies e também entre as metodologias de indução de lesão nos diferentes estudos.

\section{CONCLUSÕES}

A terapia com cartogenina, segundo protocolo utilizado, não produziu melhora nas repercussões clínicas de lesões osteocondrais induzidas e tratadas com microperfurações na tróclea lateral do fêmur em equinos.

\section{AGRADECIMENTOS}

Ao CNPq (Produtividade de Pesquisa), a FAPEMIG (Pesquisador Mineiro), a CAPES (PROEX e Bolsa de Mestrado) pelo financiamento deste estudo.

\section{REFERÊNCIAS}

BRANDT, K.D.; DIEPPE, P.; RADIAN, E. Etiopathogenesis of osteoarthritis. Med. Clin. N. Am., v.93, p.1-24, 2009.

CROWNFORD, W.H.; HOUGE, J.C.; NEIRBY D.T. et al. Pulsed radio frequency therapy of experimentally induced arthritis in ponies. Can. J. Vet. Res., v.55, p.76-85, 1991.

DENOIX, J.M.; COUDRY, V. Diagnosis of femorotibial injuries in horses. In: INTERNATIONAL CONGRESS OF WORLD EQUINE VETERINARY ASSOCIATION, 10., 2008, Moscow. Proceedings... Moscow: IVIS, 2008. p.283-289.

FRISBIE, D.D.; BOWMAN, S.M.; COLHOUN, H.A. et al. Evaluation of autologous chondrocyte transplantation via a collagen membrane in equine articular defects: results at 12 and 18 months. Osteoarthritis Cartilage, v.16, p.667$679,2008$.

GREENFIELD, G. Analytical approach to bone radiology. In: GREENFIELD, G. Radiology bone diseases. 4.ed. Philadelphia: JB Lippincott, 1986. p.1-30.

HURTIG, B.; PETER, B.F.; CECIL, E. et al. Effects of lesion size and location on equine articular cartilage repair. Can. J. Vet. Res., v.52, p.137-146, 1988.
JOHNSON, K.; ZHU S.; TREMBLAY, M.S. et al. A stem cell-based approach to cartilage repair. Science, v.336, p.717-721, 2012.

LARSEN, A.; DALE, K.; EEK, M. Radiographic evaluation of rheumatoid arthritis and related conditions by standard reference films. Acta Radiologica. v.18, p. 481-491, 1977.

MCILWRAITH, C. W. General pathobiology of the joint and response to injury. In: MCILWRAITH, C. W.; TROTTER, G. W. Joint disease in the horse. Philadelphia: W. B Saunders, 1996. p.40-70.

MCILWRAITH, C.W. Osteoarthritis (degenerative joint disease): an update. In: INTERNATIONAL CONGRESS OF THE WORLD EQUINE VETERINARY ASSOCIATION, 11., 2009, Guarujá. Proceedings... Guarujá: IVIS, 2009. p.1-5.

McILWRAITH, C.W.; FRISBIE D.D.; RODKEY W.G. et al. Evaluation of intra articular mesenchymal stem cells to augment healing of microfractured chondral defects. Arthroscopy, v.27, p.1552-1561, 2011.

MILANO, G.; DERIU, L.; SANNA PASSINO, E. et al. Repeated platelet concentrate injections enhance reparative response of microfratures in the treatment of chondral defects of the knee: an experimental study in an animal model. Arthroscopy, v.28, p.688-701, 2012.

MILANO, G.; DERIU, L.; SANNA PASSINO, E. et al. The effect of platelet rich plasma combined with microfractures on the treatment of chondral defects: an experimental study in a sheep model. Osteoarthritis Cartilage., v.8, p.971-980, 2010.

PARRY, B.W. Miscellaneous laboratory techniques. In: PRATT, P.W. Laboratory procedures for veterinary technicians. St. Louis: Mosby, 1997. p.561-626.

ROCHA JUNIOR, S. S.; MENDES, H. M. F.; BIER, S. L. et al. Avaliações macroscópica e histológica do reparo da cartilagem articular equina tratada com microperfurações do osso subcondral associadas ou não à injeção intraarticular de cartogenina. Pesq. Vet. Bras. v.36, n.4, p.272-278, 2016 
SWIDERSKI, C.E.; COOKE, E.; LINFORD, R. How to inject the medial femorotibial joint: an alternate approach. Proc. Am. Assoc. Equine Prac., v.51, [p.476-480], 2005.

WEEREN, P.R.; GRAUW, J.C. Pain in osteoarthritis. Vet. Clin. Equine, v.26, p.619-642, 2010.

XU, X.; SHEN, Y.D.; SHI, D.; JIANG, Q. Fullthickness cartilage defects repair with microfrature technique and intra: articular injection of a small molecule compound kartogenin. Sports Med. Adult Reconstr., v.22, Suppl., p.s482, 2014.
YAMADA, A.L.M.; CARVALHOI, A.M; OLIVEIRA, P.G.G. et al. Efeito do implante autólogo de plasma rico em plaquetas e células tronco mesenquimais na reparação de lesões condrais articulares induzidas experimentalmente em equinos. 2011. $145 f$.

Dissertação (Mestrado em Medicina Veterinária) - Escola de Veterinária, Universidade Júlio de Mesquita Filho, Botucatu, SP. 